\title{
COMPARAÇÃO DE DUAS TÉCNICAS DE ORQUIECTOMIA EM EQÜINOS, EMPREGADAS NO ENSINO DA TÉCNICA CIRÚRGICA VETERINÁRIA
}

\author{
Mariane Angelica Finger ${ }^{1}$, Peterson Triches Dornbusch ${ }^{1}$, Aline Fabrini Bonfá ${ }^{1}$, Lilian \\ Pamela Tapia Carreno Dornbusch ${ }^{1}$, Ivan Deconto ${ }^{1}$, Ivan Roque de Barros Filho ${ }^{1}$ \\ ${ }^{1}$ Universidade Federal do Paraná
}

Endereço para correspondência: Peterson Triches Dornbusch: petriches@ufpr.br

\begin{abstract}
RESUMO: A orquiectomia bilateral é uma cirurgia simples e rotineira na prática eqüina, embora o potencial para complicações seja alto. A complicação mais comum é a hemorragia, que se não tratada leva ao quadro de choque e morte. Diversas técnicas e variações destas estão descritas na literatura, sendo a mais freqüentemente empregada a técnica aberta. O objetivo deste estudo foi comparar duas técnicas de hemostasia aplicadas por alunos do curso de medicina veterinária, sem experiência com orquiectomia em eqüinos. Para tal foram utilizados 16 equinos machos, com idade média de seis anos $( \pm 4)$, que foram separados em dois grupos, aleatoriamente, por sorteio: GRUPO 1 , no qual os animais foram castrados utilizando-se emasculador, para promover a hemostasia $(\mathrm{n}=8)$; GRUPO 2, com animais que foram castrados utilizando-se abraçadeiras de nylon, de $2,5 \mathrm{~mm}$ de largura, para promover a hemostasia $(n=8)$. Foram mensurados os tempos do ato cirúrgico, presença de edema de prepúcio e secreção purulenta, tempo de fechamento da ferida e claudicação no pós-operatório, sendo que nenhuma diferença foi observada entre os grupos. O grupo castrado com o emasculador demonstrou maior grau de hemorragia $(P<0,05)$. As braçadeiras de nylon podem ser utilizadas na castração de eqüinos, sendo que neste experimento estas não apresentaram complicações secundárias a curto ou longo prazo. Ambas as técnicas mostraram-se viáveis e de fácil aplicabilidade na prática veterinária.
\end{abstract}

Palavras-chave: orquiectomia, eqüinos, ensino, abraçadeira de náilon.

\section{COMPARISON OF TWO TECHNIQUES IN EQUINE ORCHIECTOMY, EMPLOYED IN THE INSTRUCTION OF THE VETERINARY SURGICAL TECHNIQUE}

\begin{abstract}
The bilateral orchiectomy is a simple and routine surgery in equine practice, but the potential for complications is high. The most common complication is bleeding, which if untreated leads to cardiogenic shock and death. Several techniques and variations of this surgery are described in the literature, and the most frequently used is the open technique. The objective of this study was to compare two surgical techniques employed by students of veterinary medicine without experience with orquiectomy in horses. Sixteen male horses were used with an average age of six years $( \pm 4)$. They were divided into two groups, selected at random. In group 1 , the animals were castrated using emasculator, to promote hemostasis $(n=8)$. In group 2, animals were castrated using nylon clamp, $2.5 \mathrm{~mm}$ wide, to promote hemostasis $(n=8)$. The time of surgery, bleeding, presences of edema of the prepuce, purulent discharge in the wound, time of closing the wound and lameness after surgery were evaluated. Significant difference $(P<0,05)$ between the groups was observed just to presence of bleeding in the immediate postoperative period. The horses castrated by emasculator had a higher incidence of bleeding. The nylon clamp is safe to be used in equine orchiectomy. Both techniques were feasible and easily applicable in practice.
\end{abstract}

Key Words: orchiectomy, horses, equine practice, nylon clamp. 


\section{INTRODUÇÃO}

Há muito na história da humanidade 0 homem observou intuitivamente a relação entre 0 comportamento sexual agressivo e a presença dos órgãos sexuais, sendo que as secreções endócrinas e principalmente das gônadas têm grande influência no controle desses comportamentos. A agressividade dos machos surge na puberdade e diminui com a castração, mas pode ser restaurada com a administração de andrógenos (CABRERA, et al. 2004). Portanto a orquiectomia bilateral nos eqüinos tem a finalidade de conferir maior docilidade e favorecer o convívio em grupo e o manejo (SILVA, et al. 2006).

A supressão da secreção de hormônios acalma o animal e evita montas indesejáveis (SOUZA, 2007).

Animais inteiros podem ser mais perigosos, especialmente para pessoas menos experientes com 0 manuseamento dos animais, ainda mais em se tratando de equinos com problemas comportamentais (MARTENIUK E CARLETON, 2004).

A orquiectomia é um dos procedimentos cirúrgicos mais comumente realizados em eqüinos e apesar de ser considerada uma cirurgia simples e rotineira, o potencial para complicações é alto. Quando a intervenção é realizada por médicos veterinários habilitados, diversas técnicas cirúrgicas e anestésicas são empregadas com a finalidade de minimizar as complicações pósoperatórias e os custos com o procedimento, sobretudo quando este for realizado em cavalos de baixo potencial genético e pequeno valor comercial (SILVA, et al. 2006).

As principais complicações da orquiectomia são a hemorragia, edema escrotal, infecção incisional, peritonite, trauma peniano, e eventração nos casos de prolapso visceral através do canal inguinal (THOMAS, et al. 1998). A hemostasia durante o procedimento é um importante fator a ser considerado, pois se 0 cordão não tiver adequadamente ligado a hemorragia pode por em risco a vida do animal (MAY E MOLL, 2002).

A escolha da técnica a ser empregada muitas vezes é influenciada pela experiência pessoal, bem como a influência de determinadas escolas, na sua formação. Portanto o objetivo deste trabalho foi comparar duas técnicas de castração empregadas por alunos do curso de medicina veterinária sem experiência com orquiectomia em eqüinos.

\section{MATERIAL E MÉTODOS}

Todas as orquiectomias bilaterais foram realizadas por alunos do terceiro e quarto anos do curso de medicina veterinária de um hospital escola, sendo estes supervisionados pelo docente responsável, e com o consentimento por escrito dos proprietários. Para tal foram utilizados 16 equinos machos, sem raça definida, com idade média de seis anos $( \pm 4)$, que foram separados em dois grupos, aleatoriamente, por sorteio:

GRUPO 1: formado por oito animais, no qual foi realizada a orquiectomia bilateral, utilizando-se emasculador, para promover a hemostasia da artéria e veias testiculares.

GRUPO 2: formado por oito animais, no qual foi realizada a orquiectomia bilateral, utilizando-se abraçadeiras de nylon de $2,5 \mathrm{~mm}$ de largura, para promover a hemostasia da artéria e veias testiculares.

\section{Preparo pré-operatório}

Todos os animais foram submetidos a jejum hídrico e alimentar de doze horas, recebendo penicilina 
benzatina, na dose de $20000 \mathrm{Ul} / \mathrm{Kg}$,por quilo de peso, por via intra-muscular, imediatamente antes do procedimento cirúrgico.

\section{Procedimento anestésico}

Os animais foram sedados com acepromazina $1 \%$, na dose de $0,05 \mathrm{mg} / \mathrm{kg}$, por via intravenosa. Após quinze minutos receberam xilazina $10 \%$, na dose de $1 \mathrm{mg} / \mathrm{kg}$, associada ao butorfanol, na dose de $0,036 \mathrm{mg} / \mathrm{kg}$, também por via endovenosa. Após cinco minutos foi aplicado ropivacaína $0,2 \%$ intratesticular, num volume de $20 \mathrm{ml}$ por testículo (Figura 1). Este protocolo anestésico possibilitou a realização da intervenção com o paciente em posição quadrupedal.

\section{Procedimento cirúrgico}

Realizou-se anti-sepsia na região escrotal, com iodo povidine degermante, seguido de iodo povidine tópico. Incidiuse a pele na região distal do escroto, paralelamente a rafe mediana, estendendo a túnica dartos e a túnica vaginal, o que possibilitou a exposição do testículo. Neste momento procedeuse a separação digital entre o cremáster e o plexo vascular. O cremáster foi liberado do epidídimo na sua porção fibrosa distal. Uma pinça Carmalt curva de $24 \mathrm{~cm}$ foi aplicada no segmento mais proximal do plexo vascular possível de ser visualizado para facilitar o manuseio da estrutura. $O$ cordão espermático foi ocluído com emasculador do tipo Reimer de $24 \mathrm{~cm}$, no grupo 1 , durante 3 minutos, em seguida o plexo foi seccionado a um centímetro abaixo do local emasculado. A ligadura vascular no grupo 2 foi realizada com duas abraçadeiras de nylon de 2,5 milímetros de espessura, esterilizadas por autoclavagem, posicionadas proximalmente no cordão espermático, numa distância de um centímetro entre elas. O plex centímetro distalmente a segunda abraçadeira. A incisão permaneceu aberta para cicatrização por segunda intenção, em ambos os grupos. Repetiuse o procedimento no testículo contralateral.

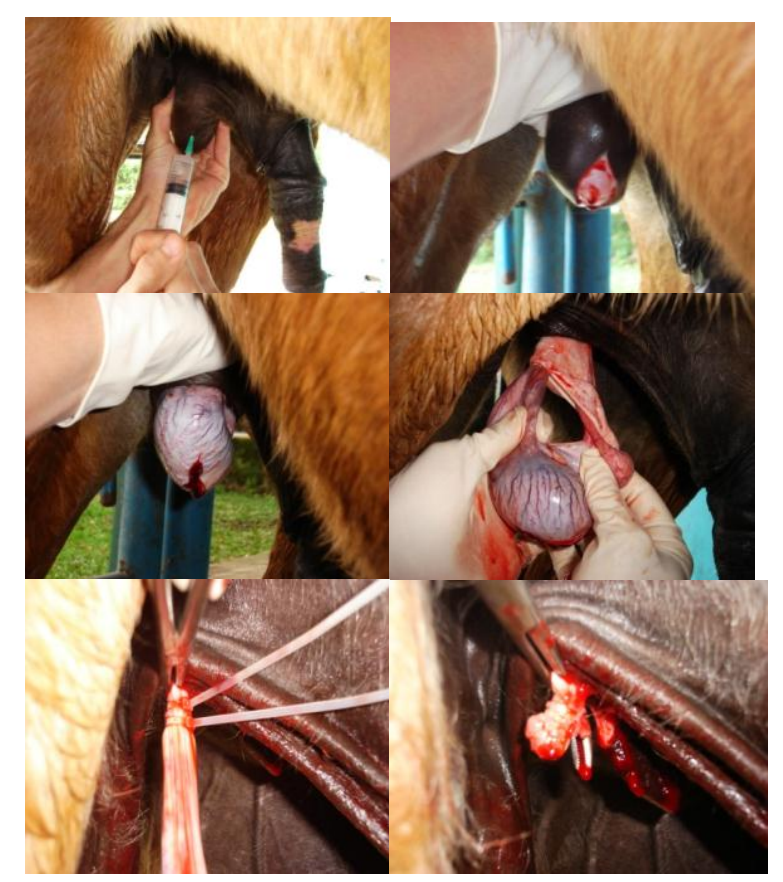

Figura 1 - Sequência da castração em estação, com o uso de braçadeira de nylon, em eqüino (grupo 2). Anestesia intra-testicular (a), incisão de pele (b), exposição do testículo (c), separação do cremáster (d), aplicação das braçadeiras de nylon (e) e aspecto final do cordão espermático após a orquiectomia.

\section{Cuidados pós-operatórios}

No período pós-operatório os animais foram medicados com ivermectina intramuscular, na dose de 200 microgramas por quilo de peso, em dose única; penicilina benzatina, na dose de $20000 \mathrm{UI}$ por quilo de peso, após 48 e 96 horas após a primeira aplicação. Para analgesia, flunixim meglumine na dose de $1,1 \mathrm{mg} / \mathrm{kg}$, via intramuscular, uma vez ao dia, durante três dias. Não foram realizados curativos no local da ferida cirúrgica, para evitar contaminação iatrogênica. Os animais foram mantidos soltos em 
piquetes individuais durante todo 0 período do pós-operatório.

\section{Avaliações}

Foram mensurados os tempos do ato cirúrgico, desde a incisão de pele até secção do plexo vascular do segundo testículo. Observou a presença de sangramento no período pósoperatório imediato segundo a Tabela 1.

Tabela 1 - Graus de sangramento da ferida cirúrgica observados após orquiectomia em cavalos.

\begin{tabular}{ll}
\hline $\begin{array}{l}\text { Grau } \\
\text { sangramento }\end{array}$ & Observações \\
\hline 0 & Sem nenhum sangramento \\
1 & $\begin{array}{l}\text { Sangramento discreto de } \\
\text { pele com algumas gotas de } \\
\text { sangue } \\
\text { Sangramento moderado, } \\
\text { onde o gotejamento } \\
\text { sanguíneo perdurou por até } \\
\text { uma hora após o término da } \\
\text { castração } \\
\text { Sangramento constante que } \\
\text { perdurou entre uma e três } \\
\text { horas } \\
\text { Sangramento intenso, por } \\
\text { mais de três horas, que } \\
\text { necessitou de re- } \\
\text { intervenção cirúrgica }\end{array}$ \\
\hline
\end{tabular}

Os animais foram reavaliados semanalmente para verificar a presença de infecção, edema prepucial, claudicação e o fechamento da ferida cirúrgica, até 28 dias após a operação, por meio de inspeção direta ou de comunicação com o proprietário. Aos seis meses após o procedimento, os proprietários foram contactados por telefone para informar possíveis complicações como: edema persistente, formação de fístulas, nódulos enrijecidos na região e presença de libido.

Para análise estatística, foram utilizados o teste de Mann-Whitney para todos os dados não paramétricos e o teste t para o tempo de cirurgia, ambos com $5 \%$ de significância.

\section{RESULTADOS}

Após o procedimento cirúrgico, observou-se que o grau de sangramento apresentou diferença entre as técnicas (figura 2).

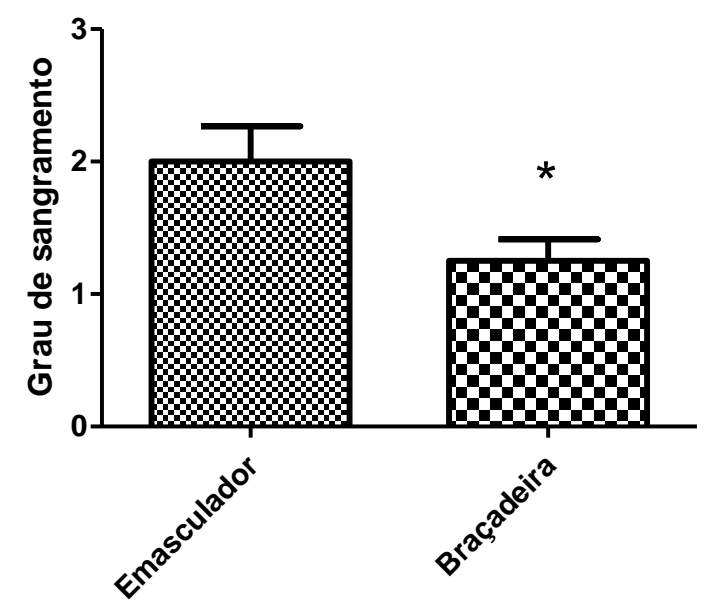

Figura 2 - Grau de sangramento após a castração, comparando-se duas técnicas cirúrgicas, onde * $p<0,05$.

As avaliações do grau de claudicação, tempo de cirurgia, presença de edema de prepúcio, presença de secreção purulenta e tempo de cicatrização final da ferida cirúrgica não apresentaram diferenças entre os grupos $(P>0,05)$. Sendo que todas estas complicações se resolveram sem a necessidade de cuidados adicionais.

Tabela 2 - Variação dos achados clínicos no período pós-operatório das castrações nos eqüinos, onde * $p<0,05$.

\begin{tabular}{|c|c|c|c|c|c|c|}
\hline $\begin{array}{l}\text { Equin } \\
o\end{array}$ & $\begin{array}{l}\text { Grau } \\
\text { de } \\
\text { sangra } \\
\text { mento }\end{array}$ & $\begin{array}{l}\text { Tem } \\
\text { po } \\
\text { de } \\
\text { cirur } \\
\text { gia } \\
\text { (min } \\
\text { utos } \\
\text { ) }\end{array}$ & $\begin{array}{l}\text { Duraç } \\
\text { ão da } \\
\text { claudi } \\
\text { cação } \\
\text { (sema } \\
\text { nas) }\end{array}$ & $\begin{array}{l}\text { Ede } \\
\text { ma } \\
\text { de } \\
\text { prep } \\
\text { úcio } \\
\text { (sem } \\
\text { anas } \\
\text { ) }\end{array}$ & $\begin{array}{l}\text { Secr } \\
\text { eção } \\
\text { purul } \\
\text { enta } \\
\text { (sem } \\
\text { anas } \\
\text { ) }\end{array}$ & $\begin{array}{l}\text { Fecha } \\
\text { mento } \\
\text { compl } \\
\text { eto da } \\
\text { ferida } \\
\text { (sema } \\
\text { nas) }\end{array}$ \\
\hline $\begin{array}{l}\text { Emasc } \\
\text { ulador } \\
(n=8)\end{array}$ & 1 a 3 & $\begin{array}{l}12 a \\
20\end{array}$ & 1 a 3 & 1 a 3 & $\begin{array}{l}1 \text { a } 2 \\
(n=2)\end{array}$ & 3 a 4 \\
\hline $\begin{array}{l}\text { Abraç } \\
\text { adeira } \\
(n=8)\end{array}$ & $1 \mathrm{a} 2^{*}$ & $\begin{array}{l}12 \mathrm{a} \\
18\end{array}$ & 1 a 3 & 1 a 3 & $\begin{array}{c}1 \text { a } 2 \\
(n=3)\end{array}$ & 3 \\
\hline
\end{tabular}


Aos seis meses após a operação os proprietários estavam completamente satisfeitos com 0 resultado do preocedimento cirúrgico e não relataram presença de complicações no local da cirurgia, nem a presença de libido nos equinos.

\section{DISCUSSÃO}

Cox (1984) já relatou em um estudo envolvendo 311 castrações que a técnica cirúrgica utilizando 0 emasculador apresentou maior taxa de sangramento, quando comparado à técnica de ligadura do cordão espermático. O que está de acordo com Silva et al (2006), que relataram um caso de óbito associado ao uso do emasculador. O uso da abraçadeira de nylon promove, além de resistência à tração, um sistema de trava eficiente por se tratar de material de fácil manuseio e esterilização, baixo custo e com boa tolerância pelo organismo de forma semelhante ao relatado por Silva et al. (2007) que utilizaram o mesmo material para ovariectomia em éguas. Neste esperimento não se optou por adicionar um grupo experimental utilizando ligaduras com fios cirúrgicos, pois na prática docente foi verificado pelos autores que acadêmicos de medicina veterinária, têm dificuldades de realizar ligaduras eficientes em vasos de grosso calibre, o que normalmente resulta em re-intervenção.

O edema é a complicação mais comum pós-castração, ocorrendo em algum grau em praticamente todos os cavalos e segundo Getman (2009), se resolve em dez a doze dias. De forma semelhante todos dezesseis cavalos operados demonstraram edema ventral na região do prepúcio, apesar da terapêutica antiinflamatória empregada e dos animais se locomoverem livremente nesta fase. $O$ fato de não haver diferenças, no que tangem ao edema, entre os grupos deve-se provavelmente ao fato de as braçadeiras de nylon serem consideradas atóxicas em um estudo conduzido por Sorbelo e colaboradores (1999), sendo considerada sua reação tecidual semelhante a do fio cirúrgico de nylon por Matos (2007).

A infecção no local é a segunda complicação mais freqüente, com uma taxa que varia de três a vinte por cento dos casos e o uso de ligaduras parece aumentar estas taxas. Neste estudo observou-se cinco casos (32\%) de presença de pus em pequena quantidade na ferida cirúrgica, entretanto não houve diferença entre as técnicas. Esta maior taxa de infecções provavelmente foi associada à falta de experiência dos acadêmicos de medicina veterinária, a operação ser realizada em condições de campo e a ferida cicatrizar por segunda intenção, o que favorece a contaminação pósoperatória, conforme citado por Getman (2009). Nenhum dos cavalos operados neste estudo evoluiu para infecção crônica, que segundo Wilson et al (2006) levam a uma significante fibrose com formação de fístulas. A taxa de complicações foi maior do que a observada por Mason et al (2005), que verificou que a castração de equinos em estação e em condições de campo apresentou $22 \%$ de complicações, enquanto cavalos castrados no centro cirúrgico, com fechamento primário da incisão apresentaram apenas $6 \%$ de complicações.

No que diz respeito a biocompatibilidade, um estudo realizado por Barros e colaboradores (2009), demonstrou que após 45 dias os lacres de poliamida estavam envoltos em uma cápsula de tecido conjuntivo composta de fibroblastos, matriz de fibras colágenas, algumas células gigantes e neovascularização. Entretanto os mesmos autores não encontraram problemas na utilização destes lacres na ovariohisterectomia de cadelas. Souza e 
Elias (2006) afirmam que os polímeros do polivinil, nylon, silicone e látex tem elevada inércia química e biológica pois seus componentes não liberam produtos tóxicos nos tecidos e nem absorvem elementos dos tecidos com que entram em contato. $O$ mesmo ocorre com alguns acrilatos (acrílicos) e com formulações de policarbonatos.

A utilização dos lacres de nylon já foi descrita e testada na medicina veterinária, desde na redução de fraturas oblíquas de fêmur de cães, por Miranda (2006), ovariohisterectomia em gatas (PAIVA et al, 2004), ovariectomia em eguas (SILVA et al, 2007; RABELO et al, 2008) e orquiectomia em eqüinos e bovinos (SILVA Et al, 2009), sendo que todos são unânimes em afirmar a praticidade, segurança e baixa taxa de complicações associadas a técnica, de forma semelhante ao observado neste estudo, podendo desta forma ser empregada na prática docente, sem incorrer em danos ao ao aprendizado.

\section{CONCLUSÃO}

As abraçadeiras de nylon podem ser utilizadas na castração de eqüinos, sendo que neste experimento estas não apresentaram complicações relevantes a curto ou em longo prazo.

Ambas as técnicas mostraram-se viáveis e de fácil aplicabilidade na prática docente, especialmente quando utilizadas por alunos com pouca habilidade cirúrgica.

A utilização do emasculador apresentou maior incidência de sangramento quando comparado a hemostasia com abraçadeiras de nylon.

\section{REFERÊNCIAS}

BARROS, B. J., SANCHES, A. W. D.; PACHALY, J. R. Utilização de abraçadeiras de náilon 6.6 (poliamida) como método de ligadura de pedículos ovarianos e coto uterino em ováriohisterectomia eletiva em cadelas (canis familiaris). Arquivos Ciências Veteterinarias Zoologia Unipar, v. 12, n. 1, p. 47-60, 2009.

CABRERA, L.; COSTA P. E. M.; FONSECA N. Ap. N. Efeito da castração pré-púbere sobre o desenvolvimento corporal de eqüinos. Acta Scientiarum Animal Sciences, v. 26, p. 273279. 2004.

COX, J. E. Castration of horses and donkeys with first intention healing. Veterinary Record; v.115, p.372-375, 1984.

GETMAN, L. M. Review of Castration Complications: Strategies for Treatment in the Field. In: American Association of Equine Practice, 55, 2009. Proceedings of 55th Annual Convention of American Association of Equine Practice, Las Vegas: 2009, p. 374-78.

MARTENIUK, J.; CARLETON, C. Castration Concerns for the Equine Owner. 2004. Disponível em: <http://www.turningpointedonkeyrescue.com/Ca stration ConcensfortheEquineOwner.pdf>. Acesso em 15/01/2011.

MASON BJ, NEWTON JR, PAYNE RJ, PILSWORTH RC. Costs and complications of equine castration: a UK practice-based study comparing standing nonsutured and recumbent sutured techniques. Equine Vetérinary Journal; v.37, p.468-72, 2005.

MATOS, W. P. Estudo comparativo das reações teciduais produzidas pela abraçadeira de náilon e o fio de náilon cirúrgico implantados na musculatura de ratas. 2007. Disponível em: <http://www.cirurgia.vet.ufba.br/uploads /2008/09/13wellington2007abracadeiraenylon.pd f> Acesso em: 07 de junho de 2009.

MAY, K. A.; MOLL, H. D. Recognition and Management of Equine Castration Complications. Compedium, v. 24, n. 2, p.11,2002.

MIRANDA, A. H.; SILVA L. A. F.; TAVARES, G. A.; AMARAL A. V. C.; MIRANDA, H. G.; Abraçadeira de náilon: resistência à tração em testes físicos e seu emprego como cerclagem 
no fêmur de cães. Ciência Animal Brasileira, v. 7, n. 3, p.299-307, 2006.

PAIVA, V. C.; RAHAL, S.; KOZU, F.O.; SETANI, M. R.; AZEVEDO, O.C. Ovariohisterectomia laparoscópica em gatas. São Paulo. In: CONGRESSO BRASILEIRO DE VIDEOCIRURGIA VETERINÁRIA 1. 2004. São Paulo. Anais 1 Congresso Brasileiro de Videocirurgia Veterinária. São Paulo, 2004. p. 40.

Rogério, R. E.; SILVA, L. A. F.; Sant'Ana, F. J. F.; Silva, M. A. M.; MOURA, M. I.; FRANCO, L. G., LIMA, C. R. O. Use of polyamide tie-rap for ovariectomy in standing mares. Acta Science Veterinarie. v. 36, n. 2.p. 119-125, 2008.

SILVA, L. A. F.; COSTA, A. C.;SOARES, L. K.; BORGES, N. C.; FERREIRA, J. L.; CARDOSOS, L. L. Orquiectomia em bovinos empregando abraçadeira de náilon na hemostasia preventiva: efeito da estação do ano, método de contenção e técnica cirúrgica. Ciência Animal Brasileira, v. 10, n. 1, p. 261270. 2009.

SILVA, L. A. F.; FRANÇA, R. O.; VIEIRA, D.; DE SOUZA, V. R.; FRANCO, L. G.; MOURA, M. I.; SILVA, M. A.; TRINDADE, B. R.; COSTA, G. L.; BERNARDES, K. A. M. Emprego da abraçadeira de náilon na orquiectomia em eqüinos. Rio Grande do Sul. Acta Scientiae Veterinariae, v. 34, p. 261-266, 2006.

SILVA, L. A. F.; FRANÇA, R. O.; VIEIRA, D.; GARCIA, A. M.; MOURA M. I.;DA SILVA M. A.; DA SILVA E. B.; TRINDADE B. R.; FRANCO, L. G. Emprego da abraçadeira de náilon, do categute e do emasculador na hemostasia preventiva de ovariectomia em éguas. Goiás. Ciência Animal Brasileira, v. 8, n. 1, p. 135146, 2007.

SORBELO, A. A.; GIODUGLI, J. N.; ANDRETTO, R. Nova alternativa para ligaduras em cirurgias videoendoscópicas ou convencionais, com emprego de fitas de nylon em estudo experimental. Revista Brasileira de Coloproctologia, v. 19, n. 1, p. 24-26. 1999.

SOUZA, N. M.; AZEVEDO, M. V.; OLIVEIRA, M. A. L.; LIMA, P. F.; ALVES, J. D. R. Castração
Da Técnica Cirúrgica Veterinária em eqüinos à campo. In: JORNADA DE ENSINO, PESQUISA E EXTENSÃO/UFRPE, 7. 2007, Recife. Anais.da Jornada de Ensino, Pesquisa e Extensão da UFRPE. Recife:, 2007, p. 3.

SOUZA, M.H., ELIAS, D. O. Materiais e Biocompatibilidade. 2006. Disponível em: <http://perfline.com/livro/download/Fdm_CEC_c ap_08.pdf> Acesso em: 15 de setembro de 2011.

THOMAS, H.; ZARUBY, J. F.; SMITH, C.; LIVESEY M. Postcastration eventration in 18 horses: the prognostic indicators for long-term survival (1985-1995). Canadian Veterinary. Journal. v. 39, p. 764-768, 1998.

WILSON, D. A.; KRAMER, J.; CONSTANTINESCU, G.; BRANSON, K. Manual of Equine Field Surgery. Missouri: Elsevier, 2006, 288p. 